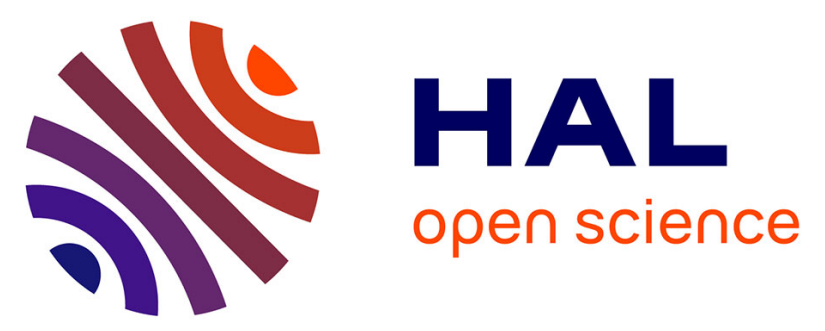

\title{
Évaluation à long terme des cystostomies continentes de type Mitrofanoff chez l'adulte : résultats à 5 ans
}

\author{
D. Poncet, B. Boillot, C. Thuillier, J.-L. Descotes, J.-J. Rambeaud, C. \\ Lanchon, J.-A. Long, G. Fiard
}

\section{- To cite this version:}

D. Poncet, B. Boillot, C. Thuillier, J.-L. Descotes, J.-J. Rambeaud, et al.. Évaluation à long terme des cystostomies continentes de type Mitrofanoff chez l'adulte : résultats à 5 ans. Progrès en Urologie, 2019, 29 (3), pp.147-155. 10.1016/j.purol.2018.12.006 . hal-02180390

\section{HAL Id: hal-02180390 \\ https://hal.science/hal-02180390}

Submitted on 22 Oct 2021

HAL is a multi-disciplinary open access archive for the deposit and dissemination of scientific research documents, whether they are published or not. The documents may come from teaching and research institutions in France or abroad, or from public or private research centers.
L'archive ouverte pluridisciplinaire HAL, est destinée au dépôt et à la diffusion de documents scientifiques de niveau recherche, publiés ou non, émanant des établissements d'enseignement et de recherche français ou étrangers, des laboratoires publics ou privés.

\section{(ㄷ)(1) $\$$}

Distributed under a Creative Commons Attribution - NonCommerciall 4.0 International 
Evaluation à long terme des cystostomies continentes de type Mitrofanoff chez l'adulte : résultats à 5 ans

D. Poncet ${ }^{a}$, B. Boillot ${ }^{a}$, C. Thuillier ${ }^{a}$, J-L Descotes ${ }^{a}, C$ Lanchon $^{a} ; J-A$ Long $^{a}$, G Fiard ${ }^{a}$

${ }^{\text {a }}$ Service d'Urologie, CHU de Grenoble BP 217, 38043 Grenoble Cedex 9 France 


\section{Introduction}

Les auto-sondages sont le gold standard pour assurer une vidange régulière de la vessie chez des patients ayant une vessie neurologique hypotonique ou spastique (1). L'accès à l'urètre peut être difficile par sa localisation pour des patients à mobilité réduite (paraplégiques, tétraplégiques), obèses, en cas d'urètres sténosés ou simplement chez la femme pour qui le déshabillage et le repérage du méat urétral restent difficiles.

La dérivation urinaire cutanée continente est alors une alternative chirurgicale aux autosondages urétraux et au Bricker.

La cystostomie continente trans-appendiculaire décrite en 1980 selon le principe de Paul Mitrofanoff (2) reste le traitement de référence. L'appendice est préféré par sa facilité de mobilisation et son diamètre qui nécessite rarement un modelage. Cette technique permet d'éviter toute résection-anastomose intestinale.

Inspirés de ce principe, Yang puis Monti ont décrit en 1997 l'utilisation d'un segment d'iléon pédiculisé de 2 à $3 \mathrm{~cm}$ de long, celui-ci sera détubulisé puis retubulisé dans le sens longitudinal (3).

Deux techniques permettent d'allonger le conduit : la réalisation d'un double Monti ou l' artifice de Casale (4) qui permet l'allongement sans double montage.

Plusieurs séries pédiatriques ont démontrées la continence de ces montages chirurgicaux sur le long terme avec des faibles taux de révisions chirurgicales (5-7).

Ces résultats sont difficilement transposables à l'adulte. En effet les vessies neurologiques adultes sont souvent des vessies avec un muscle détrusorien épais rendant plus difficile la tunnelisation du conduit en sous muqueux. Le surpoids est également plus fréquent avec nécessité de trouver des artifices pour allonger le conduit et atteindre la paroi cutanée abdominale sans tension.

Notre étude a pour objectif d'évaluer à long terme dans une population adulte, la continence, les reprises chirurgicales et la satisfaction des patients opérés d'une cystostomie continente selon Mitrofanoff.

\section{Patients et méthodes}

Les critères d'inclusions étaient la réalisation d'une chirurgie de type cystostomie continente cutanée au CHU de Grenoble entre juin 1997 et mars 2015.

Les critères d'exclusion étaient : un âge inférieur à 18 ans, un antécédent de cystostomie continente dans un autre centre et un suivi inférieur à 6 mois.

Chaque patient a été recontacté par téléphone pour répondre à un questionnaire comportant 13 questions. Le questionnaire a été élaboré selon l'échelle de Likert afin d'estimer les résultats fonctionnels. Il était évalué quantitativement : le nombre de sondages diurnes et nocturnes, la charrière (ch) des sondes utilisées, le volume mictionnel moyen.

Il était évalué qualitativement par l'échelle de Likert : la continence urinaire stomiale et urétrale, la facilité des sondages, le confort urinaire des sondages, l'impact sur la vie sociale et l'aspect esthétique de la cicatrice (Annexe 1).

\section{Analyses statistiques :}

Les réponses aux questionnaires et les complications opératoires de cette cohorte ont été colligées et analysées. Les variables quantitatives ont été décrites par la médiane et l'interquartile (25ème et 75ème percentiles), tandis que les variables qualitatives ont été décrites par la fréquence et le pourcentage. Les patients ont été stratifiés selon le type de conduit : appendico-vésicostomie ou iléo-vésicostomie. 
L'analyse statistique, réalisée en collaboration avec un statisticien a été faite grâce au test exact de Fisher à l'aide du logiciel « $\mathrm{R} »$. Les $p$ value ont été notifiées. Une valeur $p<0,05$ était considérée comme statistiquement significative.

\section{Résultats}

\section{Population initiale}

Cette étude rétrospective a inclus tous les patients opérés au CHU de Grenoble d'une cystostomie continente cutanée première.

45 patients répondaient aux critères d'inclusions (Figure 1).

L'intervention a été réalisée par 3 chirurgiens ayant opérés respectivement : 23, 12 patients et 10 patients.

Parmi les 45 patients opérés d'une cystostomie continente cutanée première, la population était composée de 21 femmes et 24 hommes.

L'âge médian était de 35 ans [22-49], l'IMC médian était de 24 [19-27].

Les caractéristiques initiales des patients sont décrites dans le tableau 1.

Les indications chirurgicales étaient variées. Il s'agissait essentiellement de vessies neurologiques $(84 \%)$, représentées par des blessés médullaires (54\%) et des patients spinabifida $(15 \%)$. Les causes non neurologiques $(16 \%)$, minoritaires, étaient majoritairement liées à une sténose urétrale $(9 \%)$. L'ensemble des étiologies des patients est résumé dans le tableau 2.

Parmi les 45 patients de la cohorte, 40 questionnaires ont été recueillis. Les questionnaires manquants concernaient : un patient décédé (après 3 ans et demi de suivi) et quatre patients injoignables.

Le suivi médian était de 64 mois [39-90].

Au terme du suivi 6 patients (13\%) n'utilisaient plus leur dérivation urinaire continente : 2 patientes sont porteuses d'un bricker (sténose complète du montage, rupture vésicale), un patient porteur d'une vessie neurologique secondaire à une toxicomanie sévère a repris des mictions normales urétrales, 3 patients ont repris les auto-sondages urétraux.

Ces auto-sondages urétraux étaient repris pour les raisons suivantes :

Le premier patient a présenté une sténose complète du conduit après deux années d'usage.

Le second patient présentait des fuites urinaires invalidantes par la stomie, malgré une réfection du trajet anti reflux et deux injections de macroplastique dans le conduit. Il a préféré reprendre les sondages urétraux.

Le troisième patient jugeait plus confortable la réalisation des sondages urétraux et a abandonné volontairement la dérivation urinaire cutanée.

\section{Technique opératoire}

Technique chirurgicale :

L'ensemble des patients ont été opérés par voie ouverte, l'appendice a été utilisé s'il admettait des sondes Ch 12 ou Ch 14. En l'absence d'appendice ou en cas de calibre inférieur à Ch 12, un tube iléal remodelé selon Monti a été réalisé.

Dans notre étude la longueur fonctionnelle minimale du conduit était de $3 \mathrm{~cm}$ mais n'excédait jamais $4 \mathrm{~cm}$. Le conduit utilisé devait être le plus court et le plus direct possible.

Le conduit a été implanté dans la vessie selon la technique anti-reflux d'implantation vésico urétérale de type Cohen.

Le trajet anti reflux a été réalisé sur deux centimètres au minium. En cas d'agrandissement vésical associé, le conduit était réimplanté sur un segment de vessie préservée. 
Si le conduit ne permettait de relier la vessie à la peau mais à la zone aponévrotique uniquement ; une plastie cutanée en fosse iliaque était réalisée pour allonger artificiellement le conduit.

Le montage chirurgical réalisé était une appendicovésicostomie (67\%) ou un segment iléal remodelé (33\%). De manière concomittante, il a été réalisé un agrandissement vésical (32 cas), ou une cæcostomie (7 cas).

La durée opératoire médiane, tous gestes confondus était de 220 minutes [120-450].

La cystostomie urinaire était localisée au niveau de l'ombilic dans $71 \%$ des cas.

Il a été réalisé 15 plasties : 4 plasties en VQZ selon Ransley (8), 5 plasties en V, et 6 plasties en VRP flap (9).

Protocole post opératoire :

Un lavage vésical était réalisé 2 à 3 fois par 24 h en cas d'agrandissement vésical afin d'éliminer le mucus.

En l'absence d'agrandissement vésical : la sonde urétrale était retirée dès le $5^{\text {ème }}$ jour. En cas d'agrandissement vésical associé, la sonde urétrale était maintenue 21 jours.

Les patients étaient sortants avec une sonde dans l'orifice de cystostomie. Une réhospitalisation était programmée au $21^{\text {ème }}$ jour post opératoire afin de procéder à l'ablation de la sonde de cystostomie et débuter l'apprentissage des auto-sondages.

La durée d'hospitalisation médiane était de 13 jours [4-59].

Le premier sondage par l'orifice de cystostomie était réalisé par le médecin. Il vérifie la facilité et la qualité du trajet, le patient est ensuite éduqué par l'équipe soignante.

\section{Continence urinaire}

La continence urinaire stomiale était définie par l'absence totale de fuites par l'orifice de cystostomie.

Au terme du suivi, $88 \%$ des patients sont continents, $77 \%$ n'ont jamais eus de fuites. Les reprises chirurgicales pour incontinence stomiales représentaient $15,5 \%$ des ré-interventions. L'incontinence urinaire stomiale était traitée par : injection de macroplastique dans le conduit (4), reprise chirurgicale par abord local (3), reprise complète du montage anti reflux (1), injection intra détrusorienne de botox (1).

La continence urinaire stomiale était équivalente quel que soit le montage chirurgical ( $\mathrm{p}=$ $0,35)$.

La continence urétrale à long terme est de $89 \%$. Dans la cohorte, 16 ré-interventions ont été réalisées : injection de toxine botulique intra détrusorienne (9), injection de macroplastique dans le sphincter (3), pose d'une bandelette TVT (1), pose d'une bandelette sous urétrale chez l'homme (1), pose d'un sphincter artificiel (1), fermeture du col vésical (1).

\section{Reprises chirurgicales}

Complications post opératoire immédiates

La morbidité post opératoire immédiate, toutes complications confondues a concerné 18 patients sur 45 (40\%) (Tableau 3). 15 patients ont présenté des complications « mineures » de stade I et II selon la classification de Clavien-Dindo. Ces complications étaient représentées par des syndromes occlusifs, des hyperthermies non étiquetées, des infections urinaires, une hématurie avec lavages vésicaux et un saignement local de la cystostomie.

Seuls trois patients ont présenté une complication de stade $\geq$ III, transfusion de 3 culots globulaires (1), collection abcédée responsable d'une éviscération (1), fistule urinaire avec montée de sondes urétérales bilatérales (1). 
$\underline{\text { Reprises chirurgicales tardives }}$

58 ré-interventions ont été répertoriées

Les reprises chirurgicales ont été réalisées chez 29 patients sur 45 (64,5\%). La reprise chirurgicale était unique chez 12 patients, deux reprises chirurgicales chez 9 patients, plus de 2 reprises chez 8 patients.

Les causes de ré-interventions étaient les suivantes : sténose de l'orifice stomial (31\%), incontinence urinaire urétrale $(29 \%)$, incontinence urinaire stomiale $(15,5 \%)$, lithiases vésicales (9\%) (Tableau 4.).

La sténose stomiale a été la complication la plus commune. Elle a concerné $31 \%$ des patients opérés. Les rétrécissements ou sténoses stomiales ont été traités par 2 dilatations au bloc opératoire et 16 reprises locales. L'incidence de sténose était plus importante en cas d'implantation ombilicale de la stomie (37\%) qu'abdominale (27\%), mais cette différence était non significative.

Les ré-interventions étaient principalement réalisées par abord local (31\%) ou endoscopique $(35 \%)$.

Seules trois interventions ont été réalisées en urgence : une péritonite secondaire à une rupture de vessie (4,9 ans après la cystostomie continente), un abcès de paroi et un hématome profond post vesicotomie pour macrolithiases.

Aucune différence significative en termes de complications tardives n'a été mise en évidence selon le type de conduit et l'association à un agrandissement vésical.

Délai des reprises chirurgicales

Le délai médian à la première reprise chirurgicale était de 7,2 mois [1,5-91].

$78 \%$ des révisions sont survenues pendant les 2 premières années. Le taux de ré-interventions décroit avec les années après un pic initial la première année à $59 \%$, puis $19 \%$ la seconde année.

Analyse du délai de survie avant survenue de la première reprise chirurgicale selon le type de montage.

L'analyse de survie a été menée au moyen du modèle de Cox.

Le délai de survie avant la survenue de la première reprise chirurgicale est identique entre les deux types de conduit utilisé (Figure 2).

\section{Satisfaction}

\section{Facilité de sondage}

Le nombre de sondages médian était de 5 en journée [2-10] et 0 la nuit [6]. Les sondages sont facilement réalisés chez $97 \%$ des patients. Aucune différence statistique n'est mise en évidence concernant la facilité de sondage selon le montage réalisé $(\mathrm{p}=0,44)$.

Estimation de la qualité de vie - Confort urinaire

94\% des patients avaient un confort urinaire très satisfaisant. Ce confort urinaire a eu un impact positif sur leur vie sociale dans $89 \%$ des cas. Un seul patient a estimé que sa vie sociale s'était dégradée. Ce patient, blessé médullaire urinait antérieurement par percussion. La dégradation de sa qualité de vie était liée à la réalisation des auto-sondages et à la quantité de matériel nécessaire pour les réaliser. 
Esthétique

Il était demandé au patient de juger de l'aspect esthétique de leur cicatrice. Celle-ci était considérée comme : très esthétique $(54 \%)$, esthétique (17\%), peu esthétique $(17 \%)$, pas du tout esthétique (6\%), sans intérêt $(6 \%)$.

\section{Discussion}

Les résultats de notre étude confirment les données de la littérature : la continence urinaire stomiale à long terme des dérivations cutanées est excellente. De nombreuses séries ont été publiées sur ce sujet notamment en pédiatrie avec des taux de continence stomiale urinaire supérieurs à $80 \%$ (5-7). Chez l'adulte, ces taux varient de $65 \%$ (10) à $86 \%$ (11) et $91 \%$ (12). Avec une continence de $88 \%$, les résultats de notre étude sont cohérents.

Bien que les techniques chirurgicales aient utilisé plusieurs types de conduits, il semblerait que seule la longueur fonctionnelle du conduit soit un facteur déterminant pour la continence. En effet, Watson (11) a mené une étude prospective sur 21 enfants opérés d'une dérivation urinaire continente cutanée. Un bilan urodynamique a été réalisé en pré et post-opératoire de manière systématique. La longueur fonctionnelle médiane des montages continents était de $3,4 \mathrm{~cm}$ contre $1,8 \mathrm{~cm}$ pour les montages non continents. Le bilan urodynamique a confirmé cette continence clinique avec des pressions de clôture deux fois plus élevées pour les conduits mesurant plus de $2 \mathrm{~cm}$. Cette longueur fonctionnelle minimale est nécessaire pour permettre une pression de clôture suffisante et assurer l'étanchéité du conduit.

Dans notre étude, ce principe était respecté avec une longueur fonctionnelle minimale de $3 \mathrm{~cm}$, mais n'excédant pas $4 \mathrm{~cm}$. À l'inverse, certaines équipes réalisent des montages avec des conduits longs, voire très longs en vue d'une meilleure continence. Cependant ces conduits longs, aux trajets moins directs sont plus difficilement cathétérisables et donc plus à risques de fausses routes.

La complication la plus fréquente est la sténose cutanée stomiale ; avec $31 \%$ des reprises chirurgicales. Ces résultats sont en accord avec les séries publiées : $31 \%$ et $36 \%$ de sténose $(9,13)$. Des techniques de plastie cutanée ont été développées pour diminuer ces taux de sténoses stomiales. Ces plasties diminuent la tension des anastomoses et amélioreraient la vascularisation du conduit. Plusieurs études ont montré une tendance en faveur de la technique VQZ. Cette technique semble diminuer le taux de sténose de la cystostomie, mais sans différence statistiquement significative (15-17).

L'ombilic a été le site historique privilégié de la cystostomie urinaire par rapport au reste de la paroi abdominale. Plusieurs études ont comparé les taux de sténoses entre ces deux localisations. La localisation de la cystostomie (ombilic ou fosse iliaque droite) n'est pas un facteur prédictif de sténose (18).

Depuis 5 ans sont apparus des bouchons siliconés en forme de clou qui sont placés dans l'orifice de cystostomie entre les sondages. L'utilisation de ces clous a permis de diminuer le taux de sténoses des cæcostomies chez l'enfant à court terme (suivi médian 12 mois) (19). Cependant, aucune étude n'a évalué l'efficacité de ces « clous siliconés » pour les dérivations urinaires..

Il est fréquent dans la population des patients spina-bifida de présenter d'importantes fuites urétrales liées à des troubles sphinctériens. Certaines équipes préconisent une fermeture systématique du col vésical. Dans notre série, un seul patient a bénéficié d'une fermeture du col vésical associée au geste de dérivation urinaire continente. Devant la persistance des fuites, il a ensuite été réalisé : une injection de macroplastique dans le col vésical, suivi de 
l'implantation d'un sphincter artificiel. La fermeture du col vésical en deuxième intention n'a concerné qu'un patient sur 45. D'ailleurs les séries publiées chez les spina-bifida rapportent des taux variables de fermeture du col vésical allant de $17 \%$ (15) à $71 \%$ (19), suggérant une attitude chirurgicale propre à chaque service. Dans notre centre, le maintien de l'accès urétral est privilégié pour deux raisons.

Tout d'abord, l'accès urétral reste une voie d'accès facile pour la réalisation des fibroscopies vésicales et autres gestes endoscopiques.

Enfin, le maintien de cet accès permet toujours la réalisation de sondages urétraux en cas de difficulté de sondage par le Mitrofanoff (rétrécissement, sténose).

La laparoscopie et la robotique se sont considérablement imposées cette dernière décade comme abord mini invasif. Plusieurs séries chez l'enfant ont comparé le robot à la chirurgie ouverte dans la confection des dérivations urinaires cutanées continentes. À ce jour, la plus grande étude pédiatrique comparant la voie ouverte $(n=28)$ à la voie robotique $(n=39)$ menée par Grimsby ne rapporte aucune différence en matière de continence urinaire stomiale finale (20). Le taux global de complications chirurgicales est également semblable.

Cependant, les complications chirurgicales du robot étaient plus précoces (260 jours versus 862 jours $p=0,037$ ) et plus graves (Clavien III). La nature des complications était également différente : en chirurgie ouverte la sténose de la stomie restait la cause la plus fréquente, alors que l'incontinence stomiale était la complication la plus fréquente en chirurgie robotique.

L'équipe de Piechaud a décrit la première réalisation robotique intra-corporelle d'un double montage de Monti associé à un agrandissement vésical chez l'adulte (21). Cette équipe a ensuite publié la première série de 15 cas chez l'adulte, opérés par laparoscopie simple $(\mathrm{n}=$ 11) ou robot assisté $(n=4)$. Plusieurs séries confirment la faisabilité et la fiabilité du geste chirurgical (21).

La laparoscopie favorise la récupération post opératoire, diminue la douleur et améliore l'aspect esthétique. Cependant à ces avantages s'opposent des difficultés techniques avec des temps opératoires initiaux plus longs. La durée opératoire médiane était de 150 minutes pour la confection d'un mitrofanoff trans-appendiculaire, 225 minutes avec agrandissement concomitant et enfin 270 minutes en cas de montage selon Monti couplé à agrandissement. Les pertes sanguines sont estimées à $140 \mathrm{~mL}$, avec une durée d'hospitalisation médiane de 14 jours.

Dans notre série, la majorité des complications a eu lieu la 1ère année comme l'ont décrit l'équipe de Thomas et celle de Leslie [2,3]. Après ce pic initial, les complications ont tendance à diminuer avec les années. Cependant même à très long terme, le risque de reprise chirurgicale persiste comme le démontre l'étude de Liard (5), avec un suivi moyen de 20 ans [15-23]. Il est encore trop tôt pour comparer les taux de complications entre un abord chirurgical ouvert et un abord laparoscopique. En effet dans ces dernières séries les suivis médians sont courts de l'ordre de 22 mois (21)

Dans notre cohorte, $87 \%$ des cystostomies continentes étaient pérennes après un suivi médian de 64 mois [39-90].

Nous avons évoqué l'amélioration de la qualité de vie globale mais celle-ci est étroitement liée à la qualité de vie sexuelle. Une réadaptation sexuelle satisfaisante après cystostomie continente est liée à deux facteurs déterminants : l'acquisition d'une autonomie pour la continence urinaire et la préservation de l'image corporelle. L'absence de fuites urinaires lors des rapports sexuels joue un rôle prépondérant selon les patients de la série de Moreno (24). 
D'autres facteurs liés à l'environnement sont décrits par Bouassida (25) tels que le statut matrimonial et la présence d'une partenaire compréhensive facilitant la stabilité morale, psychologique et sociale.

En dépit des ré-interventions nécessaires, le taux de satisfaction reste élevé et corrélé à une amélioration globale de la qualité de vie.

Les principales limites de notre étude sont, le caractère rétrospectif de l'étude et l'utilisation d'un questionnaire non validé. Un suivi clinique par auto questionnaire semblait approprié car la gravité des symptômes présentés par les patients n'est pas corrélée à leur impact sur la qualité de vie. Cependant seul un questionnaire de qualité de vie, validé en langue française, rempli avant et après la chirurgie aurait permis d'attester d'un effet bénéfique de l'intervention sur la qualité de vie des patients.

Nous avons choisi de stratifier notre cohorte en deux groupes selon le type de montage chirurgical (Mitrofanoff ou Monti). Aucune analyse n'est revenue significative en matière de complications ou de continence. Il est impossible de conclure à l'absence réelle de différence. Un recueil de données multicentriques permettrait d'augmenter la puissance de l'étude, mais il faudrait alors prendre en compte un biais de confusion lié aux pratiques chirurgicales propres à chaque centre.

Notre étude malgré son caractère rétrospectif et une cohorte de 45 patients, reste une série importante dans une population adulte.

\section{Conclusions}

La dérivation cutanée continente urinaire reste une alternative aux auto-sondages urétraux, continente et pérenne à très long terme, au prix de ré-interventions multiples, dont le patient doit être informé.

\section{Déclaration d'intérêts}

Les auteurs déclarent ne pas avoir de conflit d'intérêts en relation avec cet article.

\section{Remerciements}

Ce travail est soutenu par l'Association Spina Bifida et Handicaps ASBH.

Un prix a été obtenu en 2016. 
Bibliographie

1. Di Benedetto P. Clean intermittent self-catheterization in neuro-urology. Eur J Phys Rehabil Med. 2011 Dec;47(4):651-9.

2. Mitrofanoff P. [Trans-appendicular continent cystostomy in the management of the neurogenic bladder]. Chir Pédiatrique. 1980;21(4):297-305.

3. Monti PR, Lara RC, Dutra MA, de Carvalho JR. New techniques for construction of efferent conduits based on the mitrofanoff principle. Urology. 1997 Jan 1;49(1):112-5.

4. Casale AJ. A Long continent ileovesicostomy using a single piece of bowel. J Urol. 1999 Nov 1;162(5):1743-5.

5. Liard A, Séguier-Lipszyc E, Mathiot A, Mitrofanoff P. the mitrofanoff procedure: 20 years later. J Urol. 2001 Jun;165(6, Supplement):2394-8.

6. Kaefer M, Tobin MS, Hendren WH, Bauer SB, Peters CA, Atala A, et al. Continent urinary diversion: the Children's Hospital experience. J Urol. 1997 Apr;157(4):1394-9.

7. King LR. Continent urinary diversion in children: the American experience. Scand J Urol Nephrol Suppl. 1992;142:85-6.

8. P. R. Operative Pediatric Urology (2nd ed.). pp. 109-114. (Churchill Livingstone, Philadelphia (2001)).

9. Franc-Guimond J, González R. Simplified Technique to Create a Concealed

Catheterizable Stoma: The VR Flap. J Urol. 2006 Mar;175(3):1088-91.

10. Chabchoub K, Ketata H, Fakhfakh H, Bahloul A, Mhiri MN. Dérivation urinaire de Mitrofanoff. Mécanismes physiques et explication urodynamique de la continence. Prog En Urol. 2008 Feb;18(2):120-4.

11. Watson SH, Bauer SB, Peters CA, Mandell J, Colodny AH, Atala A, et al. Comparative Urodynamics of Appendiceal and Ureteral Mitrofanoff Conduits in Children. $\mathbf{J}$ Urol. 1995 Aug;154(2):878-82.

12. Malone PS, Ransley PG, Kiely EM. Preliminary report: the antegrade continence enema. The Lancet. 1990 Nov 17;336(8725):1217-8.

13. Bruyère F, Sotto A, Escaravage L, Cariou G, Mignard J-P, Coloby P, et al. Recommandations de bonnes pratiques cliniques : l'antibioprophylaxie en chirurgie urologique, par le Comité d'infectiologie de l'association française d'urologie (CIAFU). Prog En Urol. 2010 Feb;20(2):101-8.

14. McAndrew HF, Malone PSJ. Continent catheterizable conduits: Which stoma, which conduit and which reservoir? BJU Int. 2002;89(1):86-9.

15. De Ganck J, Everaert K, Van Laecke E, Oosterlinck W, Hoebeke P. A high easy-totreat complication rate is the price for a continent stoma. BJU Int. 2002 Aug 1;90(3):240-3.

16. Gowda BDR, Agrawal V, Harrison SCW. The continent, catheterizable abdominal conduit in adult urological practice. BJU Int. 2008 Dec 1;102(11):1688-92.

17. Redshaw JD, Elliott SP, Rosenstein DI, Erickson BA, Presson AP, Conti SL, et al. Procedures Needed to Maintain Functionality of Adult Continent Catheterizable Channels: A Comparison of Continent Cutaneous Ileal Cecocystoplasty with Tunneled Catheterizable Channels. J Urol. 2014 Sep;192(3):821-6.

18. P.-H. Savoie, A. Van Hove, G. Olagui, J. Laroche, P. Riviere, L. Lemesle, R. Fournier. Dérivations urinaires continentes cutanées. EMC Elsevier Masson Tech Chir. 2012 Février;41-214.

19. Bihrle R, Klee LW, Adams MC, Foster RS. Early clinical experience with the transverse colon-gastric tube continent urinary reservoir. J Urol. 1991 Sep;146(3):751-3. 20. Grimsby GM, Jacobs MA, Gargollo PC. Comparison of Complications of RobotAssisted Laparoscopic and Open Appendicovesicostomy in Children. J Urol. 2015 
Sep;194(3):772-6.

21. Rey D, Oderda M, El Helou E, Robbiani J, Lopez L, Piechaud P-T. Feasibility of Robotic Double Yang-Monti Ileal Conduit With Bladder Augmentation: Surgical Technique. Urology. 2013 Aug 1;82(2):480-4.

22. Leslie B, Lorenzo AJ, Moore K, Farhat WA, Bägli DJ, Pippi Salle JL. Long-term followup and time to event outcome analysis of continent catheterizable channels. J Urol. 2011 Jun;185(6):2298-302.

23. Thomas JC, Dietrich MS, Trusler L, DeMarco RT, Pope JC, Brock JW, et al. Continent Catheterizable Channels and the Timing of Their Complications. J Urol. 2006 Oct 1;176(4):1816-20.

24. Moreno JG, Chancellor MB, Karasick S, King S, Abdill CK, Rivas DA. Improved quality of life and sexuality with continent urinary diversion in quadriplegic women with umbilical stoma. Arch Phys Med Rehabil. 1995 Aug 1;76(8):758-62.

25. Bouassida M, Smaoui W, Fourati M, Mseddi MA, Chabchoub K, Chaabouni A, et al. Étude de la sexualité masculine après dérivation urinaire externe continente de type Mitrofanoff. Prog En Urol. 2016 Feb 1;26(2):115-20. 
$\underline{\text { Tableau 1. Caractéristiques cliniques des patients au moment de la chirurgie }}$

$\underline{\text { Tableau 2. Les indications chirurgicales }}$

Tableau 3. Complications immédiates

Tableau 4. Reprises chirurgicales tardives

Figure 1. Diagramme de flux, cohorte étudiée

Figure 2. Délai de survie avant survenue de la première complication selon le type de conduit utilisé

$\underline{\text { Annexe 1. Questionnaire }}$

$\underline{\text { Table } 1 . \text { Baseline characteristics of treated patients. }}$

Table 2. Surgical choices.

Table 3. Early adverse events.

$\underline{\text { Table 4. Delayed reinterventions. }}$

Figure 1. Flow diagramm troop.

Figure 2. Overall survival according to the duct used.

Appendix 1. Survey 


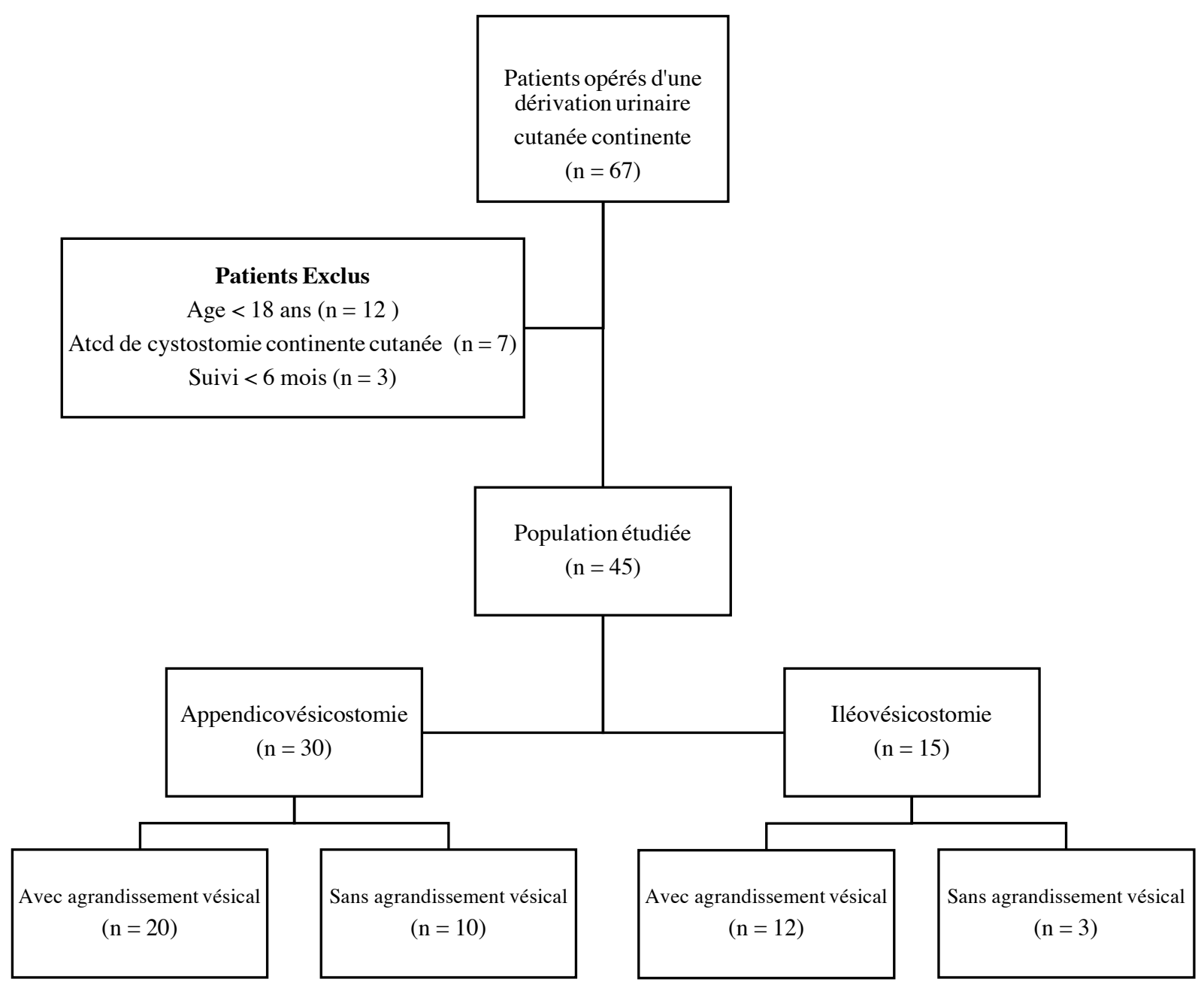




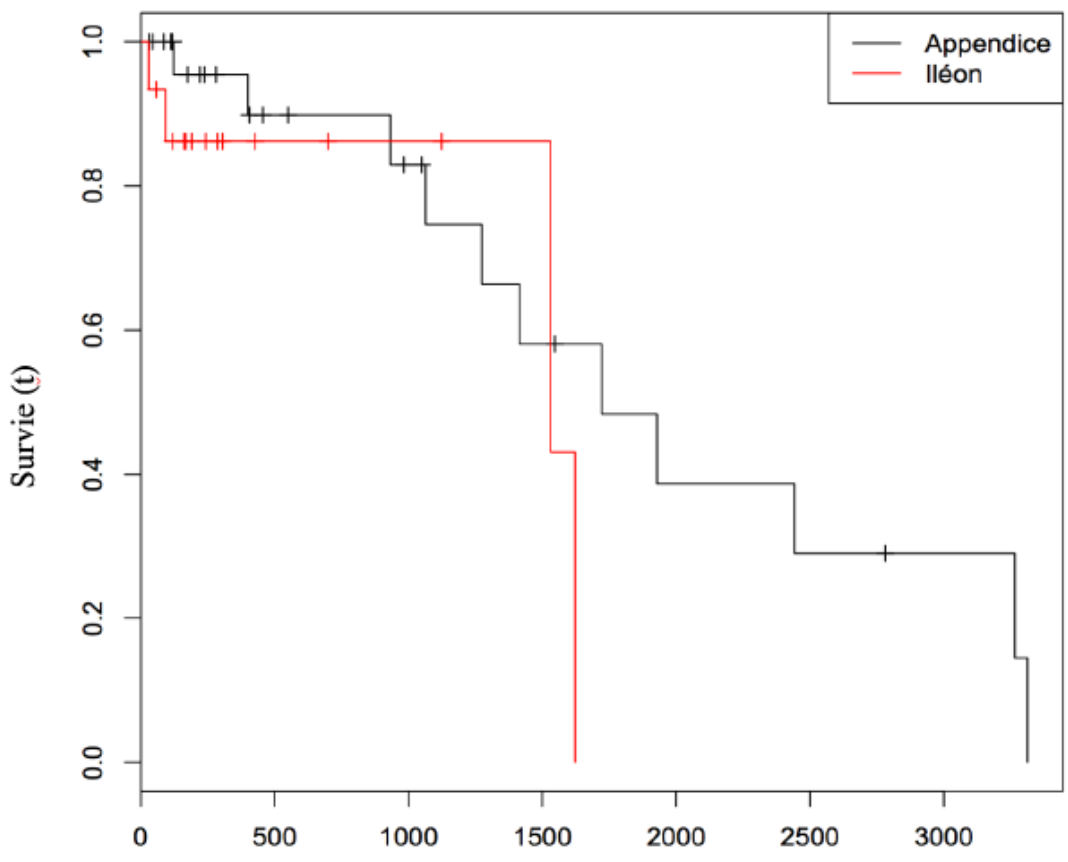

\begin{tabular}{cccccccc} 
& \multicolumn{8}{c}{ Temps (jours) } \\
Appendice & 30 & 15 & 11 & 9 & 3 & 3 & 1 \\
\hline Ileon & 15 & 5 & 3 & 2 & 1 & 0 & 0
\end{tabular} 


\begin{tabular}{|c|c|c|c|}
\hline & $\begin{array}{l}\text { Total } \\
\mathrm{n}=45\end{array}$ & $\begin{array}{l}\text { Appendicovésicostomie } \\
\qquad \mathrm{n}=30\end{array}$ & $\begin{array}{l}\text { Iléo-vésicostomie } \\
\qquad \mathrm{n}=15\end{array}$ \\
\hline \multicolumn{4}{|l|}{ Sexe } \\
\hline Femme & 21 & 14 & 7 \\
\hline Homme & 24 & 16 & 8 \\
\hline Âge médian (années) & 35 & 34 & 39 \\
\hline$\left(25^{\text {ème }}-75^{\text {ème }}\right.$ percentiles $)$ & [22-49] & {$[23-44]$} & {$[21-53]$} \\
\hline IMC médian $\left(\mathrm{kg} / \mathrm{m}^{2}\right)$ & 24 & 22 & 26 \\
\hline ( $25^{\text {ème }}-75^{\text {ème }}$ percentiles) & {$[19-27]$} & [18-26] & [22-28] \\
\hline \multicolumn{4}{|c|}{ IMC : Indice de masse corporelle } \\
\hline
\end{tabular}


$n=45$

\section{Neurologiques}

Blessés médullaires

Paraplégiques

Tétraplégiques

Spina-bifida

Méningocèles

Myéloméningocèles

Maladies auto-immunes

SEP

Lupus

Non étiquetée

Dénervation post chirurgie (sarcome)

Toxicomanie

Non neurologiques

Sténose urétrale récidivante

Post prostatectomie

Post cystoprostatectomie-néovessie

Traumatisme urètre

Uropathie malformative

Autres

Syndrome de Hinman

Incontinence urinaire 


\begin{tabular}{|ccccc|}
\hline Caractéristiques & Tous patients & $\begin{array}{c}\text { Appendico- } \\
\text { vésicostomie } \\
\mathrm{n}=30\end{array}$ & $\begin{array}{c}\text { Iléo-vésicostomie } \\
\mathrm{n}=45\end{array}$ & $\begin{array}{c}\text { Agrandissement } \\
\text { vésical } \\
\mathrm{n}=32\end{array}$ \\
\hline $\begin{array}{c}\text { Complications immédiates } \\
\text { Score de Clavien-Dindo }\end{array}$ & $\mathbf{1 8}$ & $\mathbf{1 3}$ & $\mathbf{5}$ & $\mathbf{1 4}$ \\
I & & & & \\
II & 8 & 7 & 1 & 6 \\
IIIa & 7 & 4 & 3 & 6 \\
IIIb & 1 & 1 & 0 & 2 \\
IV et V & 2 & 1 & 1 & 0 \\
\hline
\end{tabular}




\begin{tabular}{|c|c|c|c|c|}
\hline Ré-interventions tardives & $\begin{array}{c}\text { Tous } \\
\text { patients } \\
\mathrm{n}=58\end{array}$ & $\begin{array}{c}\text { Appendico- } \\
\text { vésicostomie } \\
n=30\end{array}$ & $\begin{array}{c}\text { Iléo- } \\
\text { vésicostomie } \\
\mathrm{n}=28\end{array}$ & $\begin{array}{c}\text { Agrandissement } \\
\text { vésical } \\
n=43\end{array}$ \\
\hline \multicolumn{5}{|l|}{ Vésicales } \\
\hline \multicolumn{5}{|l|}{ Lithiases } \\
\hline Lithotritie intra-vésicale & 4 & 4 & 0 & 4 \\
\hline Taille vésicale & 1 & 0 & 1 & 1 \\
\hline \multicolumn{5}{|l|}{ Fuites urétrales } \\
\hline Toxine botulique intra-détrusorienne & 9 & 2 & 7 & 5 \\
\hline Macroplastique intra sphinctérien & 3 & 2 & 1 & 3 \\
\hline Cervicopexie & 1 & 1 & 0 & 1 \\
\hline TVT & 1 & 1 & 0 & 1 \\
\hline Bandelette sous urétrale & 1 & 1 & 0 & 1 \\
\hline Sphincter artificiel & 1 & 0 & 1 & 1 \\
\hline \multicolumn{5}{|l|}{$\underline{\text { Conduit }}$} \\
\hline Reprise locale sténose & 16 & 7 & 9 & 13 \\
\hline Dilatation sténose & 2 & 1 & 1 & 1 \\
\hline Réimplantation vésicale du conduit & 3 & 1 & 2 & 2 \\
\hline Macroplastique conduit & 4 & 0 & 4 & 2 \\
\hline Réfection du montage & 4 & 3 & 1 & 2 \\
\hline Bricker & 1 & 1 & 0 & 0 \\
\hline Exploration & 3 & 3 & 0 & 2 \\
\hline \multicolumn{5}{|l|}{$\underline{\text { Autres }}$} \\
\hline Evacuation hématome profond & 1 & 0 & 1 & 1 \\
\hline Bricker suite rupture vessie & 1 & 1 & 0 & 1 \\
\hline Bloc pudendal & 1 & 1 & 0 & 1 \\
\hline Fermeture pariétale sur éviscération & 1 & 1 & 0 & 0 \\
\hline
\end{tabular}

